\title{
Exploration of 8-piperazine-1-ylmethylumbelliferone for application as a corrosion inhibitor for mild steel in hydrochloric acid solution
}

\author{
A.M. Resen, ${ }^{1}$ M.M. Hanoon, ${ }^{1}$ W.K. Alani, ${ }^{2}$ A. Kadhim, ${ }^{3}$ A.A. Mohammed, ${ }^{4}$ \\ T.S. Gaaz, ${ }^{4}$ A.A.H. Kadhum, ${ }^{5,6}$ A.A. Al-Amiery ${ }^{2,6}{ }^{(D)}$ and M.S. Takriff ${ }^{6}$ (D) \\ ${ }^{1}$ Production engineering and metallurgy department, University of Technology - Iraq, \\ Baghdad, Iraq \\ ${ }^{2}$ Energy and renewable energies technology center, University of Technology - Iraq, \\ Baghdad, Iraq \\ ${ }^{3}$ Laser \& optoelectronics engineering department, University of Technology - Iraq, \\ Baghdad, Iraq \\ ${ }^{4}$ Al-Mussaib Technical College, Al-Furat Al-Awsat Technical University, 51006 Babylon, \\ Iraq \\ ${ }^{5}$ College of Medicine, University of Al-Ameed, Karbala, Iraq \\ ${ }^{6}$ Department of Chemical Engineering, Faculty of Engineering and Build Environment, \\ Universiti Kebangsaan Malaysia, Bangi, Malaysia \\ *E-mail: dr.ahmed1975@gmail.com
}

\begin{abstract}
The name umbelliferone is from the Umbelliferae plants family, which in turn is named after the umbrella-shaped inflorescences, each of which is called an umbel. It was found in carrot, coriander, and garden angelica, mouse-ear hawkweed, and bigleaf hydrangea. In this study, a new umbelliferone derivative, namely 8-piperazine-1-ylmethylumbelliferone $(\mathbf{8 P})$, was synthesized, and its structure was characterized and confirmed by Fourier transform infrared spectroscopy and nuclear magnetic resonance spectroscopy. The potential of using $\mathbf{8 P}$ as an ecofriendly corrosion inhibitor for mild steel in hydrochloric acid solution was examined using gravimetric techniques confirmed by topology characterization through scanning electron microscopy technique. The effect of inhibitor concentration, solution temperature, and immersion time on the inhibition efficiency of $\mathbf{8 P}$ was also investigated. The results from weight loss techniques reveal that $\mathbf{8 P}$ exhibits significant anti-corrosive characteristics toward mild steel dissolution in $1 \mathrm{M}$ hydrochloric acid environment. 8P with a concentration of $0.0005 \mathrm{M}$ exhibited the highest inhibition efficiency of $93.42 \%$ at $303 \mathrm{~K}$. Scanning electron microscopy exhibited that $\mathbf{8 P}$ inhibited mild steel corrosion. The adsorption isotherm indicated that $\mathbf{8 P}$ exhibited physical and chemical sorption mechanisms and obeyed the Langmuir adsorption isotherm model. The relationship between the inhibition behavior of the $\mathbf{8 P}$ and its molecular structure was examined by density functional theory (DFT) which confirmed that $\mathbf{8 P}$ possessed significant inhibition efficiency which was consistent with experimental findings.
\end{abstract}


Keywords: 8-piperazine-1-ylmethylumbelliferone, corrosion, DFT, HOMO, Langmuir isotherm.

Received: November 26, 2020. Published: March 12, 2021

doi: $\underline{10.17675 / 2305-6894-2021-10-1-21}$

\section{Introduction}

Coumarins have been considered as an important class of heterocyclic molecules due to their common medicinal and biological applications [1]. Coumarins exhibited considerable biological activities such as antitumor, antimicrobial, and anticoagulant activities [2]. These observations led us to assess the corrosion inhibition features of 8-piperazine-1ylmethylumbelliferone (8P) as a newly synthesized coumarin. 8P has been selected in this study as a novel corrosion inhibitor due to its molecular formula that has tertiary and secondary amines besides hydroxy and carbonyl groups and possesses one $\mathrm{O}$ atom in the ring, in addition to $\pi$-electrons, through which they can easily bind to the mild steel surface and increase the inhibition efficiency [3]. The characteristic feature for the synthesis of $\mathbf{8 P}$ is that it is a one-pot reaction providing a high yield [4]. In the present effort, the density functional theory (DFT), as a theoretical technique, was used to understand the mechanism of corrosion inhibition of the synthesized compound in comparison with conventional techniques, and help stimulate the experimental findings with calculated molecular characteristics such as the energy of the highest occupied molecular orbital (HOMO), energy of the lowest unoccupied molecular orbital (LUMO), energy gap $(\Delta E)$, electronegativity $(\chi)$, softness $(\sigma)$, hardness $(\eta)$ and fraction of transferred electrons $(\Delta N)$ from $\mathbf{8 P}$ to mild steel. This technique is cheap and time-saving [5-8]. In a joint work of the Malaysian National University (Malaysia) with the University of Technology (Iraq), some novel coumarins that exhibit corrosion inhibition efficiency of $83 \%$ to $97 \%$ at $0.005 \mathrm{M}$ were previously reported [9-17]. Herein, we have synthesized a novel corrosion inhibitor $\mathbf{8 P}$ to enhance the inhibitive performance compared to that of the previously published coumarins at lower concentrations because this one has more hetero atoms than in the previous work on coumarins. Comparison of the inhibitory efficacy of $\mathbf{8 P}$ with other recently published inhibitors confirms that the current inhibitor has better corrosion inhibition performance. Gupta et al. have published a synthesis of new corrosion inhibitors derived from $\alpha$-aminophosphonate for mild steel, and they found that the inhibition efficiencies were 58-96\% at concentrations of 141-564 ppm. On the other hand, Wang et al. published a synthesis of $S$-benzyl-O, $O^{\prime}$-bis(2-naphthyl)dithiophosphate derivatives as corrosion inhibitors for mild steel, which demonstrated inhibition efficiencies of 70-95\% at concentrations of 40-100 $\mathrm{mg} / \mathrm{L}[18,19]$. To the best of our knowledge, there is no previous study that uses $\mathbf{8 P}$ as a corrosion inhibitor of mild steel in a hydrochloric acid solution. This work presents an investigation of a new eco-friendly synthesized coumarin for the corrosion inhibition of mild steel coupons in hydrochloric acid solution through weight loss techniques. The surface topology studies of mild steel coupons in the absence and presence of $\mathbf{8 P}$ were used to characterize the protective inhibitor layer. 
DFT Da was also conducted to study the correlations between the experimental findings and the quantum chemical values.

\section{Experimental Section}

\subsection{Materials}

The starting materials used in this investigation were provided by Sigma/Aldrich in Selangor, Malaysia. The composition of the mild steel specimen is as follows (wt.\%): carbon, 0.210; manganese 0.050; silicon 0.380; aluminum 0.010; sulfur 0.050; phosphorus 0.090 ; balance iron. Mild steel pieces were mechanically cut into $4.5 \times 2.5 \times 0.025 \mathrm{~cm}$ specimens for gravimetric techniques. The surface of the mild steel specimens was abraded with emery papers and washed with acetone and double distilled water. A 37\% hydrochloric acid solution of analytical grade was diluted with double distilled water to prepare the $1 \mathrm{M}$ hydrochloric acid test solution.

\subsection{Inhibitor}

8P was synthesized by refluxing a solution of piperazine $(0.01 \mathrm{~mol})$ in absolute ethyl alcohol $(70 \mathrm{~mL})$ with formalin $(37 \%, 0.02 \mathrm{~mol})$ for $1 \mathrm{~h}$ at $60^{\circ} \mathrm{C}$. An ethanolic solution of umbelliferon $(0.01 \mathrm{~mol})$ was added and refluxing was continued for $12 \mathrm{~h}$. The precipitate was recrystallized from $\mathrm{CH}_{2} \mathrm{Cl}_{2}$. Yield $45 \%$, m.p. $233^{\circ} \mathrm{C}$. The target compound (Scheme 1) was characterized by NMR, FTIR, and CHN techniques. Nuclear magnetic resonance spectroscopy (NMR) was conducted with an AVANCE III $600 \mathrm{MHz}$ spectrometer (Bruker, Billerica, Massachusetts, United States of America), with dimethyl sulfoxide as the internal standard. The delta values were expressed in ppm. Fourier-transform infrared spectroscopy (FTIR) was conducted with a Shimadzu 8300 spectrometer. CHN elemental analysis was carried out with a Carlo Erba $5500 \mathrm{CHN}$ elemental analyzer. FT-IR $\left(\mathrm{cm}^{-1}\right)$ : 3413.8 for O$\mathrm{H}, 3097.4$ for $\mathrm{N}-\mathrm{H}), 17328.9$ for $\mathrm{C}=\mathrm{O}$ lactone; ${ }^{1} \mathrm{H}$ NMR, DMSO-d $\mathrm{d}_{6}, \delta$, ppm: $2.57(4 \mathrm{H}, \mathrm{d}$, $\left.\mathrm{CH}_{2}\right), 2.89\left(4 \mathrm{H}, \mathrm{d}, \mathrm{CH}_{2}\right), 3.99\left(2 \mathrm{H}, \mathrm{s}, \mathrm{N}-\mathrm{CH}_{2}\right), 6.33(1 \mathrm{H}, \mathrm{d},=\mathrm{CH}), 6.88(1 \mathrm{H}, \mathrm{d},=\mathrm{CH}), 7.29$ $(1 \mathrm{H}, \mathrm{d}), 7.30(1 \mathrm{H}, \mathrm{d})$, and $7.44(1 \mathrm{H}, \mathrm{d}) .{ }^{13} \mathrm{CNMR}, \mathrm{DMSOd}_{6}, \delta$, ppm: 161.8, 159.7, 153.8, $144.9,130.2,126.5,114.8,113.2,109.7,55.3,54.1,52.6$ and 44.9. Elemental analysis $\left(\mathrm{C}_{14} \mathrm{H}_{16} \mathrm{~N}_{2} \mathrm{O}_{3}\right)$ : C, 64.60 (64.76); H, 6.20 (6.62); N, 10.76 (10.59).

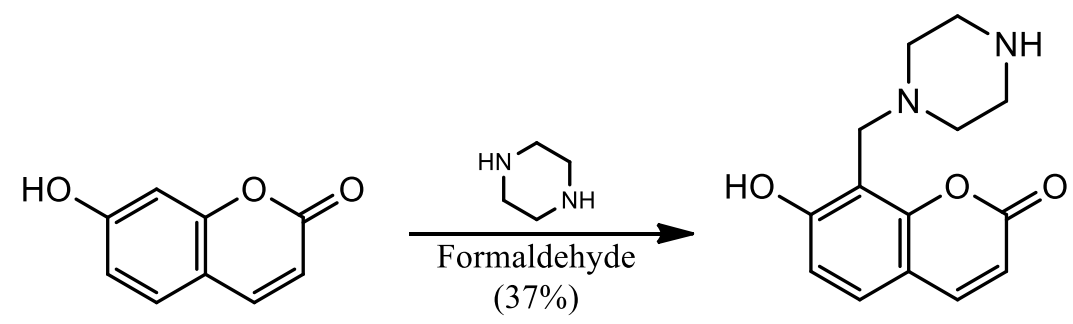

Scheme 1. Synthetic route for the preparation of $\mathbf{8 P}$. 


\subsection{Weight Loss Techniques}

Weight loss measurements were conducted with mild steel specimens by exposing them in $1 \mathrm{M}$ hydrochloric acid without and with the addition of $\mathbf{8 P}$ at various concentrations $(0.0001-0.0005 \mathrm{M})$. The mild steel specimen was taken out after the immersion period (1, $5,10,24 \mathrm{~h})$, washed, dried, and weighed accurately. The corrosion rate $\left(C_{\mathrm{R}}\right)$ was evaluated using equation (1):

$$
C_{\mathrm{R}}(\mathrm{mm} / \text { year })=\frac{87.6 \mathrm{~W}}{a t D}
$$

where $W$ is weight loss, $a$ is surface area of the mild steel coupon, $t$ is exposure time, and $D$ is the density of the mild steel $\left(\mathrm{g} \cdot \mathrm{cm}^{-3}\right)$.

\subsection{Scanning Electron Microscopy}

Surface morphology investigations were conducted on mild steel specimens after exposure in hydrochloric acid solution in the absence and presence of $\mathbf{8 P}$ at the optimum concentration $(0.0005 \mathrm{M})$. A Scanning electron microscope TM1000 Hitachi Tabletop Microscope was utilized for the analyses.

\subsection{Quantum Chemical Calculations}

Quantum chemical calculations using the density functional theory (DFT) method were conducted for the 8P molecule. The $6-31+\mathrm{G}(\mathrm{d}, \mathrm{p})$ basis set was selected for the calculations which were conducted with Gaussian 09 software. The quantum chemical parameters were determined to estimate the electronic parameters of the most stable conformers of the inhibitor molecule. The energy of the highest occupied molecular orbital ( $\left.E_{\mathrm{HOMO}}\right)$ and the energy of lowest unoccupied molecular orbital $\left(E_{\mathrm{LUMO}}\right)$ as the energies of frontier molecular orbital (FMO), energy gap $(\Delta E)$, global hardness $(\eta)$, softness $(\sigma)$, absolute electronegativity $(\chi)$, and a number of transferred electrons $(\Delta N)$ were calculated using equations $(2-6)$ $[20,21]$.

$$
\begin{gathered}
\Delta E=E_{\text {HOMO }}-E_{\mathrm{LUMO}} \\
\eta=-\left(\frac{E_{\mathrm{HOMO}}-E_{\mathrm{LUMO}}}{2}\right) \\
\sigma=\frac{1}{\eta} \\
\chi=-\left(\frac{E_{\mathrm{HOMO}}-E_{\mathrm{LUMO}}}{2}\right) \\
\Delta N=\frac{\chi_{\mathrm{Fe}}-\chi_{\mathrm{inh}}}{2\left(\eta_{\mathrm{Fe}}+\eta_{\mathrm{inh}}\right)}
\end{gathered}
$$

where $\chi_{\mathrm{Fe}}$ and $\eta_{\mathrm{Fe}}$ were $7 \mathrm{eV} / \mathrm{mol}$ and $0 \mathrm{eV} / \mathrm{mol}$, respectively. 


\section{Results and Discussion}

\subsection{Weight Loss Measurements. Effect of Inhibitor Concentration}

The experimental findings obtained from gravimetric techniques, such as the corrosion rate and inhibition efficiency, are demonstrated in Figures 1-6, which exhibits that in the presence of various concentrations of $\mathbf{8 P}$ for immersion periods $1,5,10 \mathrm{~h}$, the corrosion rate decreases and the inhibition efficiency increases. At $24 \mathrm{~h}$ immersion time the corrosion rate increases and the inhibition efficiency decreases.

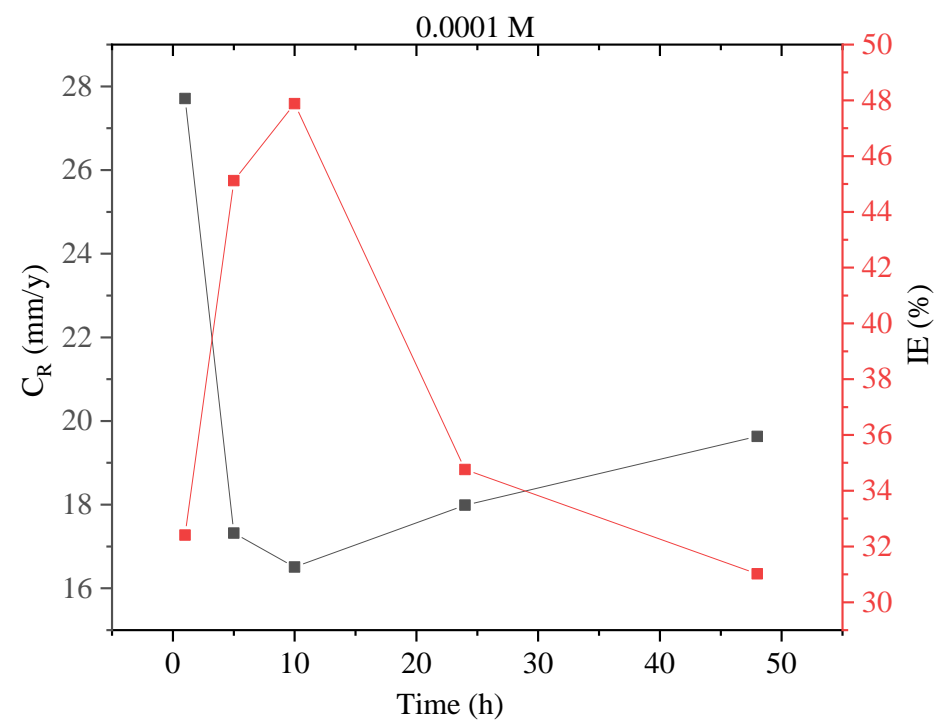

Figure 1. Gravimetric measurements for mild steel in the absence and presence of $0.0001 \mathrm{M}$ $\mathbf{8 P}$ in $1 \mathrm{M}$ hydrochloric acid solution at $303 \mathrm{~K}$.

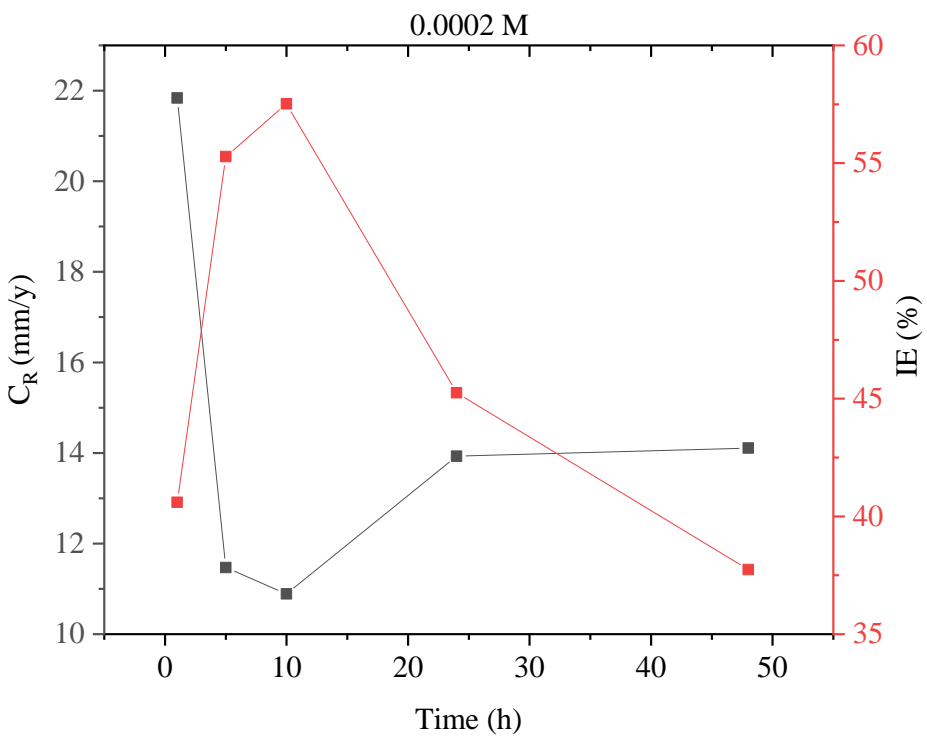

Figure 2. Gravimetric measurements for mild steel in the absence and presence of $0.0002 \mathrm{M}$ $\mathbf{8 P}$ in $1 \mathrm{M}$ hydrochloric acid solution at $303 \mathrm{~K}$. 


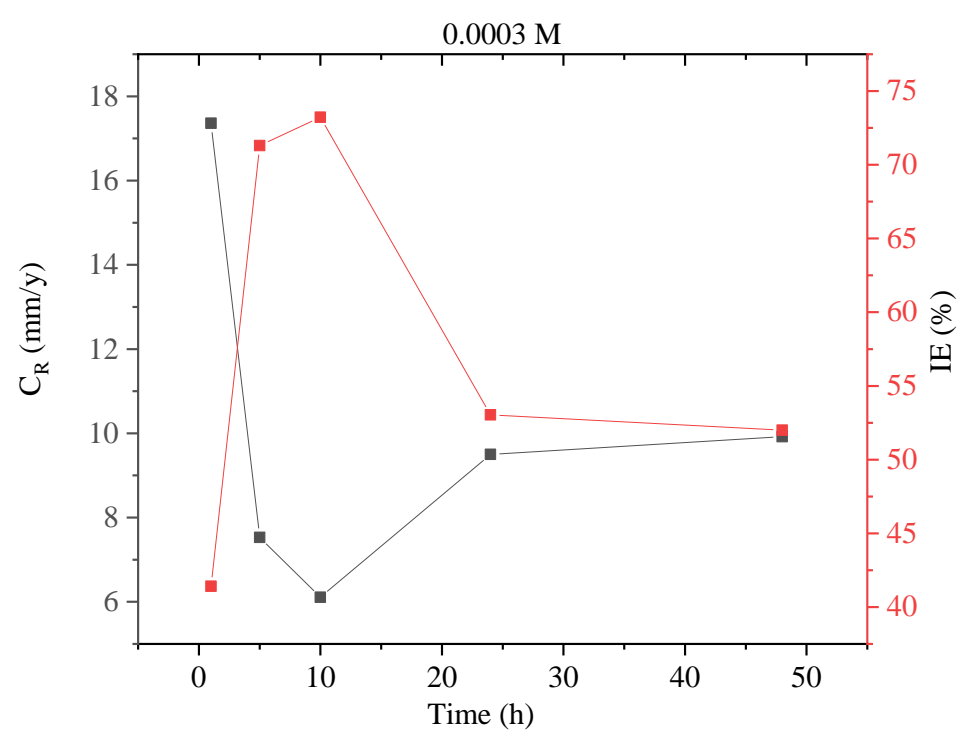

Figure 3. Gravimetric measurements for mild steel in the absence and presence of $0.0003 \mathrm{M}$ $\mathbf{8 P}$ in $1 \mathrm{M}$ hydrochloric acid solution at $303 \mathrm{~K}$.

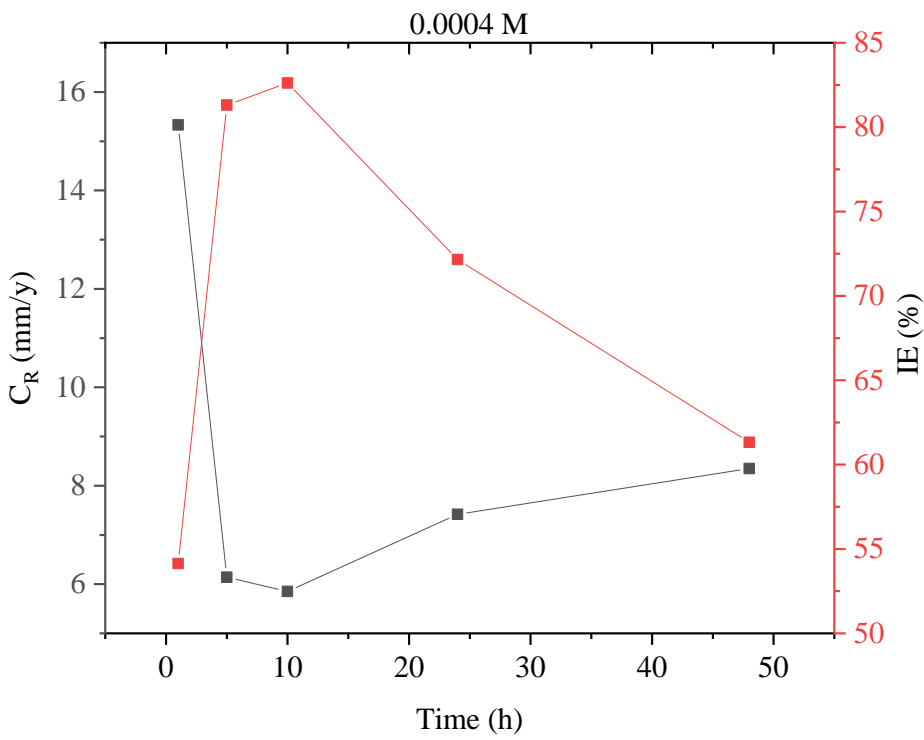

Figure 4. Gravimetric measurements for mild steel in the absence and presence of $0.0004 \mathrm{M}$ $\mathbf{8 P}$ in $1 \mathrm{M}$ hydrochloric acid solution at $303 \mathrm{~K}$.

This elucidates that the corrosion inhibitive performance of the investigated compound is concentration-dependent [22] and this is reflected in the inhibition effectiveness plot versus concentration as demonstrated in Figures $1-5$. The gravimetric technique is a reliable method to optimize the inhibitor concentration effect on corrosive inhibitive performance [23]. 


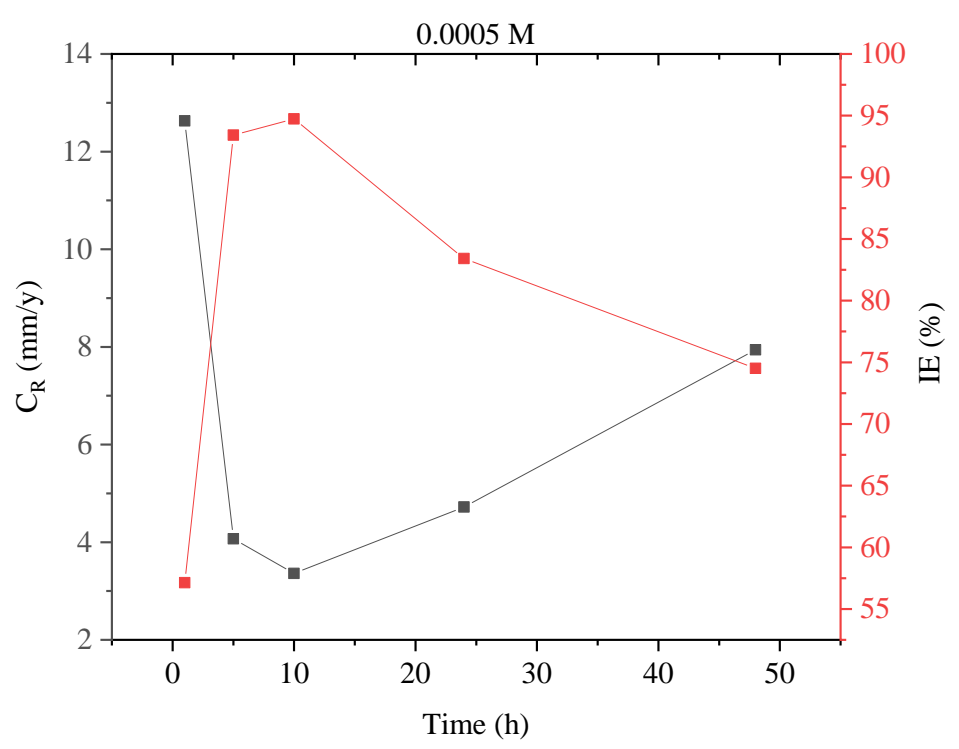

Figure 5. Gravimetric measurements for mild steel in the absence and presence of $0.0005 \mathrm{M}$ $\mathbf{8 P}$ in $1 \mathrm{M}$ hydrochloric acid solution at $303 \mathrm{~K}$.

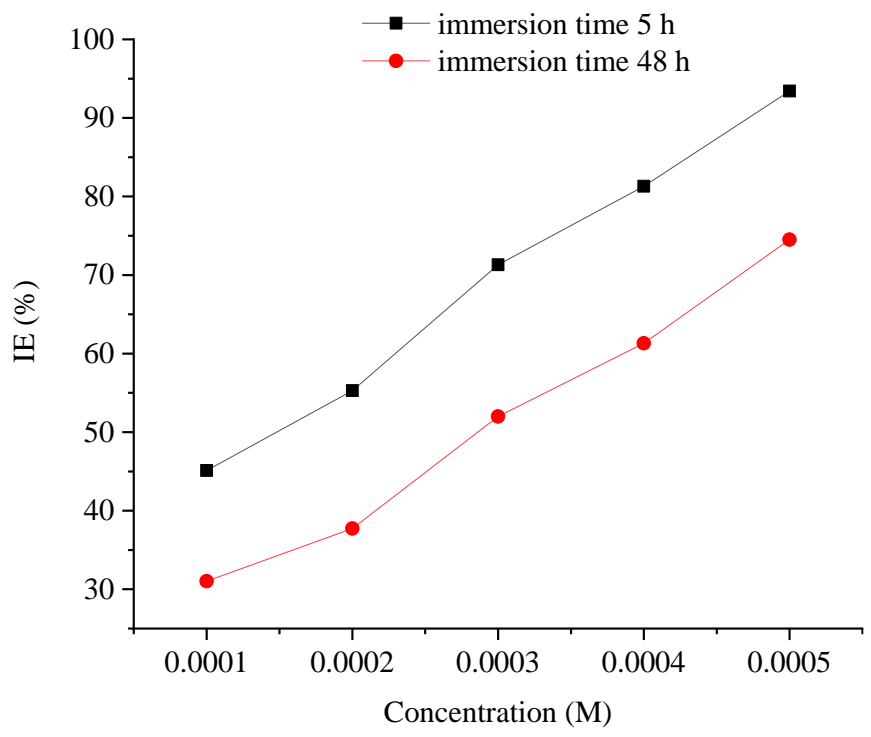

Figure 6. A comparison between the effect of different concentrations of $\mathbf{8 P}$ on the inhibition efficiency for mild steel in $1 \mathrm{M}$ hydrochloric acid solution at 5 and $48 \mathrm{~h}$ immersion times.

The inhibitive performance of the investigated $\mathbf{8 P}$ was studied for mild steel in $1 \mathrm{M}$ hydrochloric acid utilizing various concentrations of the inhibitor $(0.0001-0.0005 \mathrm{M})$ and different immersion times of 1, 2, 5, 10, and $24 \mathrm{~h}$. The inhibition efficiency increased sharply with an increase in the concentration of the studied inhibitor. The efficiency remained almost constant at the optimum immersion time (between 5 and $10 \mathrm{~h}$ ) and the inhibition efficiency of the $\mathbf{8 P}$ at $0.0005 \mathrm{M}$ was $93.4 \%$ for $5 \mathrm{~h}$ immersion time. The inhibition efficiency $(\eta, \%)$ was evaluated according to Equation 7: 


$$
\eta=\frac{C_{\mathrm{R}}-C_{\mathrm{R}(\mathrm{i})}}{C_{\mathrm{R}}} \times 100
$$

where $C_{\mathrm{R}}$ is the corrosion rate $\left(\mathrm{mg} \cdot \mathrm{cm}^{-2} \cdot \mathrm{h}^{-1}\right)$ in the absence of $\mathbf{8 P}$ and $C_{\mathrm{R}(\mathrm{i})}$ is the corrosion rate in the presence of $\mathbf{8 P}$.

The investigated inhibitor controls the corrosion through the surface coverage $(\theta)$ of mild steel surface which increases the inhibition efficiency. The relationship between inhibition efficiency and surface coverage can be calculated according to Equation 8:

$$
\theta=\frac{\eta}{100}
$$

The increase in $\mathbf{8 P}$ concentration led to an increase in the surface coverage and adsorption extent due to the presence of many inhibitor molecules, which leads to increased inhibition efficiency. The high inhibition efficiency of the studied compound is attributed to the presence of many heteroatoms and the presence of aromatic rings as well as double bonds and thus an increase in the inhibition efficiency.

\subsection{Effect of Temperature}

The effect of temperature $(308-338 \mathrm{~K})$ on the corrosion inhibition efficiency for mild steel at the optimum concentration is demonstrated in Figures 7-11. It is obvious from the plots that the inhibition efficiency of $\mathbf{8 P}$ decreases with an increase in temperature. This is due to the desorption of adsorbed $\mathbf{8 P}$ molecules from the surface of mild steel at a higher temperature of the acidic solutions.

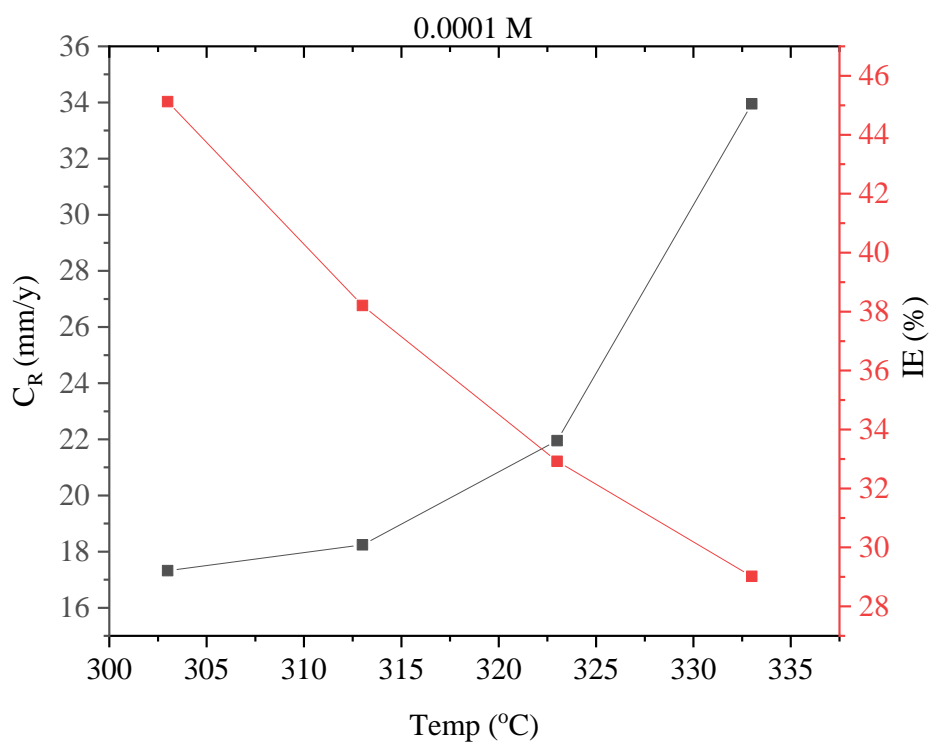

Figure 7. Effect of temperature $(308-338 \mathrm{~K})$ on the inhibitive efficiency for mild steel in the presence of $0.0001 \mathrm{M}$ of $\mathbf{8 P}$ in $1 \mathrm{M}$ hydrochloric acid solution. 


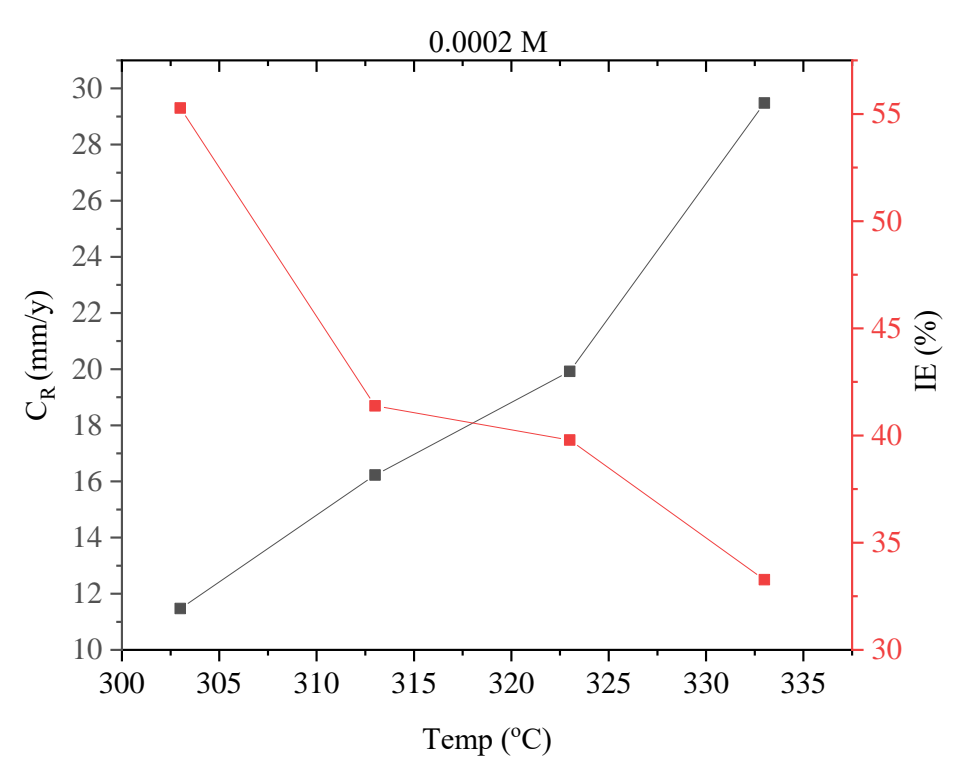

Figure 8. Effect of temperature (308-338 K) on the inhibitive efficiency for mild steel in the presence of $0.0002 \mathrm{M}$ of $\mathbf{8 P}$ in $1 \mathrm{M}$ hydrochloric acid solution.

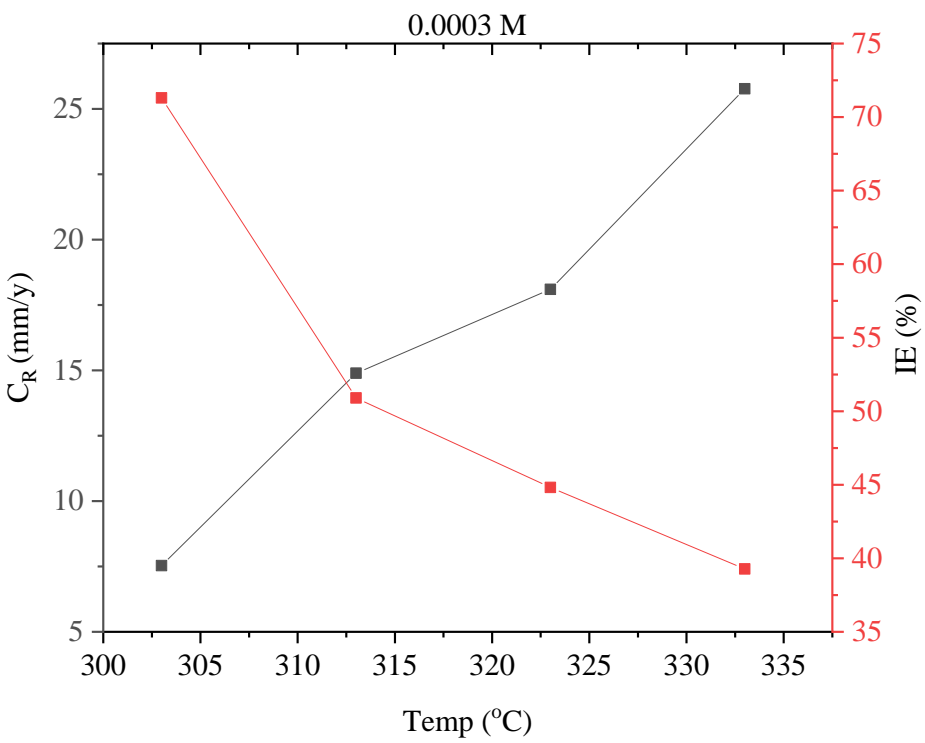

Figure 9. Effect of temperature (308-338 K) on the inhibitive efficiency for mild steel in the presence of $0.0003 \mathrm{M}$ of $\mathbf{8 P}$ in $1 \mathrm{M}$ hydrochloric acid solution.

\subsection{Adsorption Isotherm}

To understand the mode of interactions between the inhibitor molecules and the metal surface, adsorption isotherms were studied. Inhibitor molecules that are adsorbed on a mild steel surface never attain the actual equilibrium but tend to attain steady state adsorption. When the rate of corrosion is reduced upon addition of an inhibitor, the process of adsorption tends to reach a quasi-equilibrium state. The quasi-equilibrium adsorption nature of inhibitor molecules can be studied utilizing the proper adsorption isotherm. The experimental data 
were fitted to different adsorption models, and the plots are demonstrated in Figures 12-14. The main equations (9-11) of the adsorption isotherms are listed accordingly.

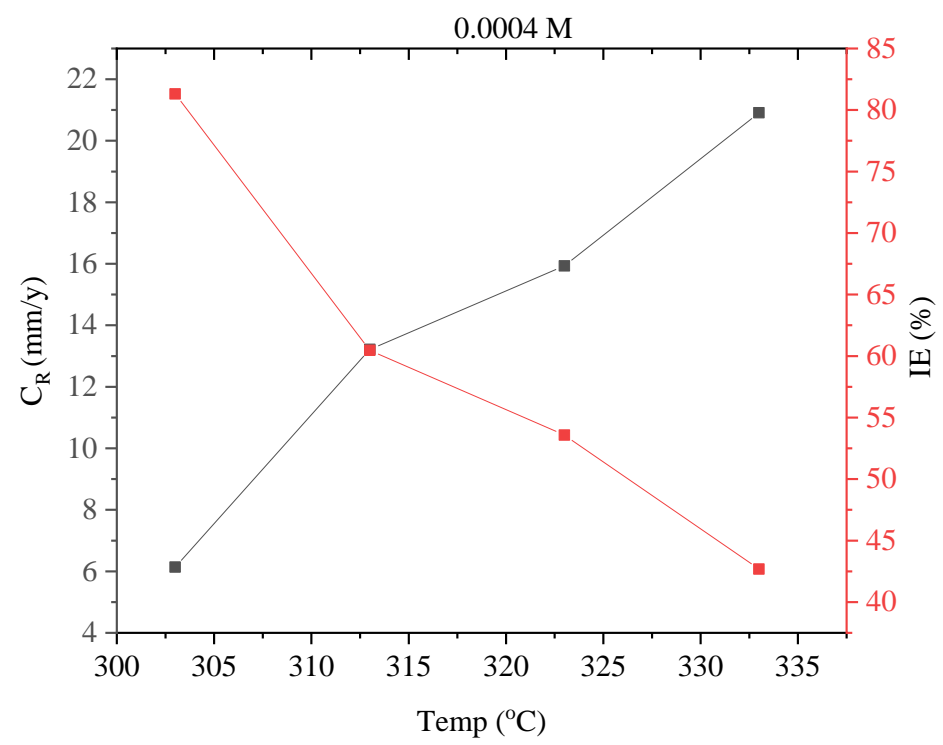

Figure 10. Effect of temperature $(308-338 \mathrm{~K})$ on the inhibitive efficiency for mild steel in the presence of $0.0004 \mathrm{M}$ of $\mathbf{8 P}$ in $1 \mathrm{M}$ hydrochloric acid solution.

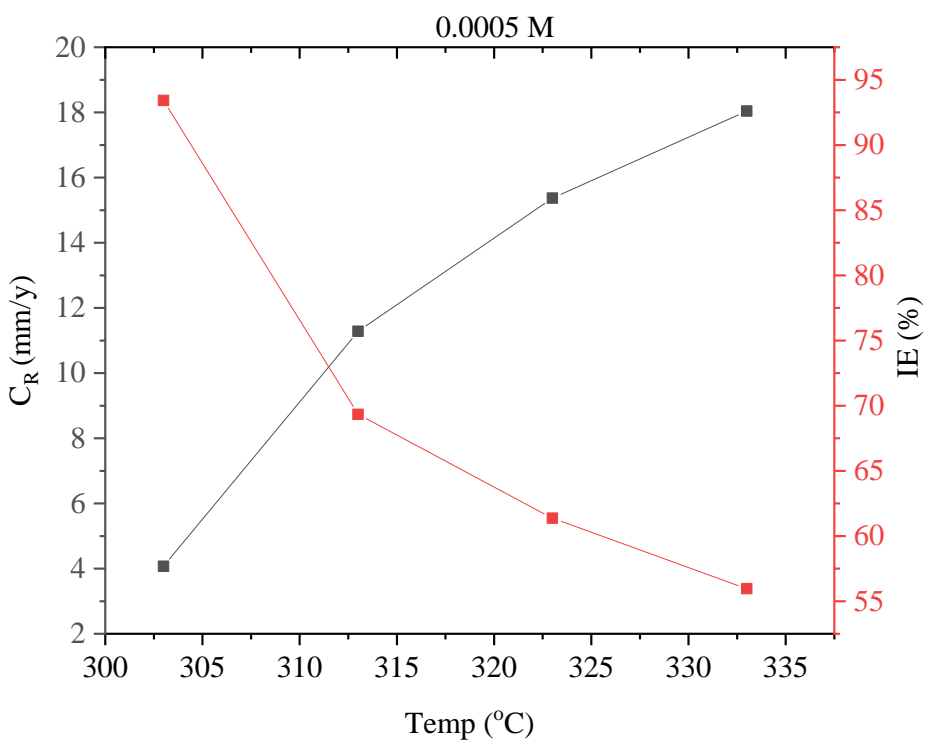

Figure 11. Effect of temperature $(308-338 \mathrm{~K})$ on the inhibitive efficiency for mild steel in the presence of $0.0005 \mathrm{M}$ of $\mathbf{8 P}$ in $1 \mathrm{M}$ hydrochloric acid solution.

Temkin isoterm:

$$
\exp ^{(\mathrm{f} \theta)}=K_{\mathrm{ads}} C
$$

Freundlich isotherm:

$$
\theta=K_{\text {ads }} C
$$


Langmuir isotherm:

$$
\frac{C}{\theta}=\frac{1}{K_{\mathrm{ads}}}+C
$$

where $K_{\text {ads }}$ is the equilibrium constant for the adsorption process and $C$ is the concentration of the corrosion inhibitor studied.

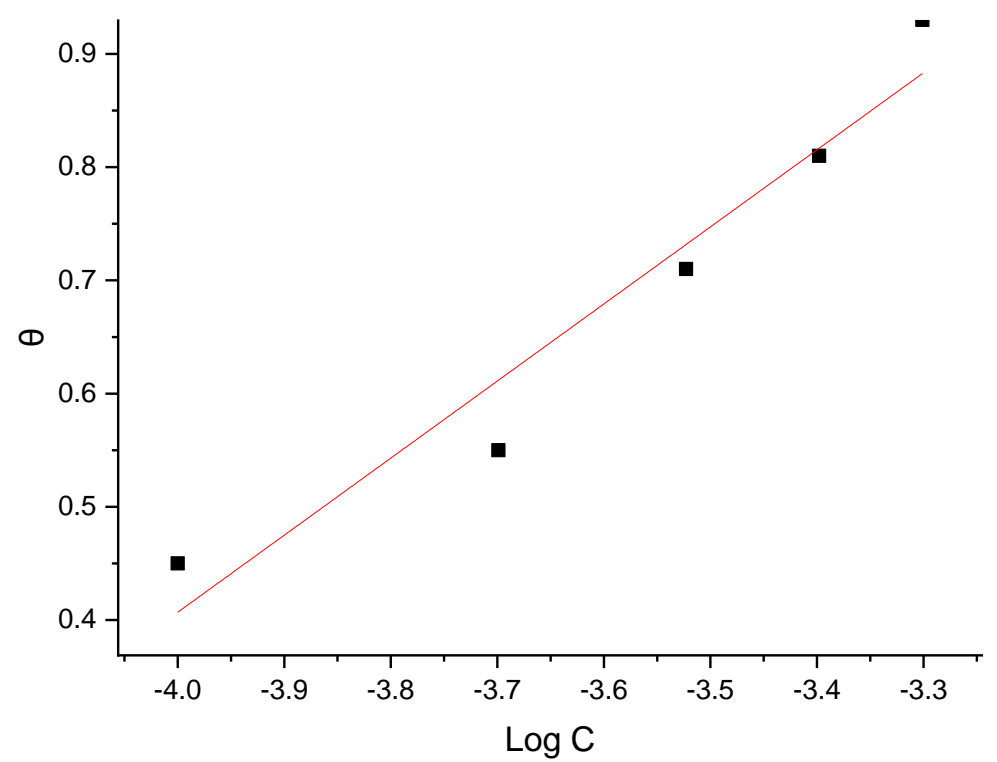

Figure 12. Temkin adsorption isotherm plot for mild steel in the presence of $\mathbf{8 P}$.

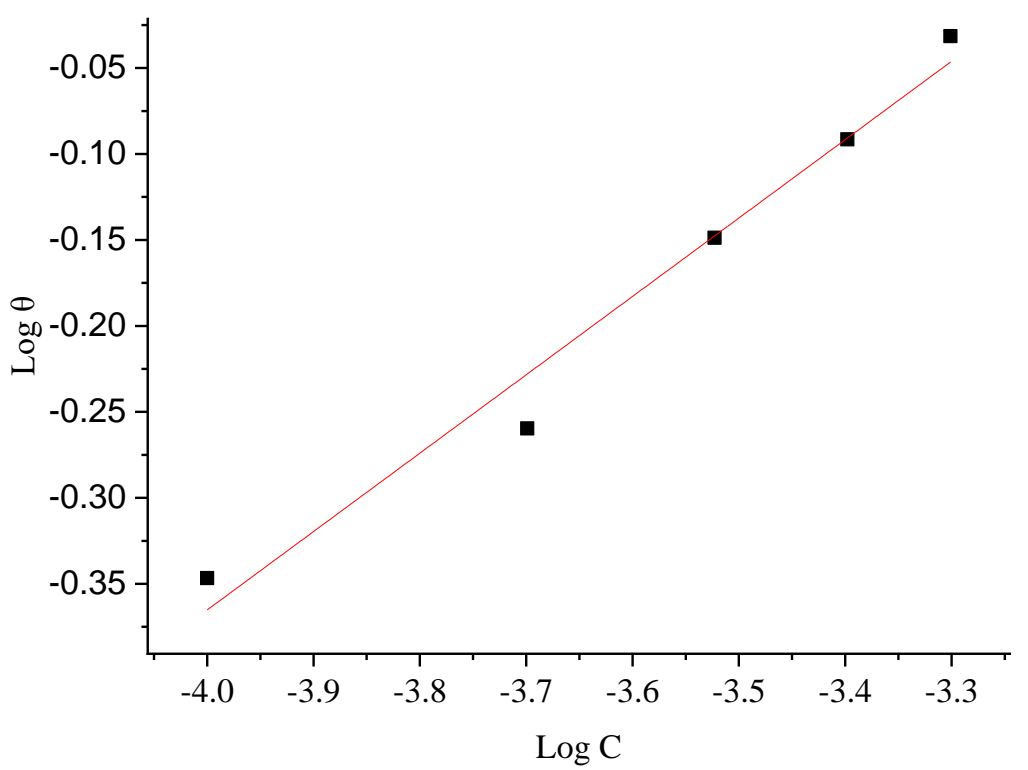

Figure 13. Freundlich adsorption isotherm plot for mild steel in the presence of $\mathbf{8 P}$. 


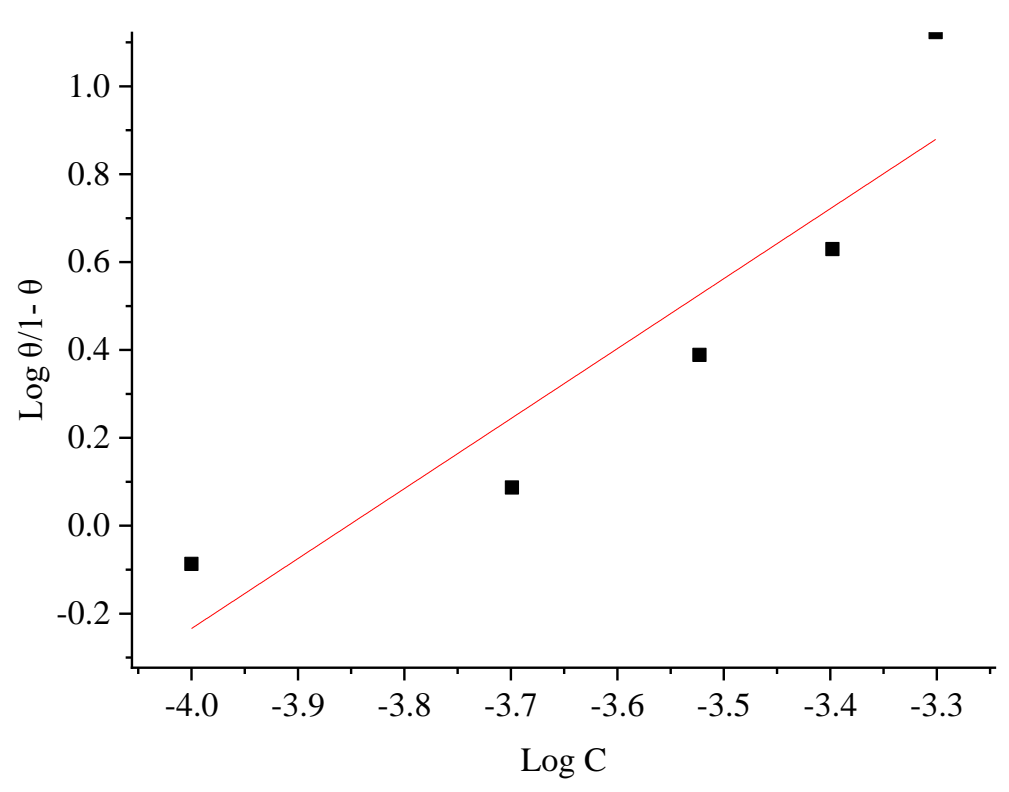

Figure 14. Langmuir adsorption isotherm plot for mild steel in the presence of $\mathbf{8 P}$.

It can be seen from the plot $(\log (\theta / 1-\theta) v s . \log C)$ that the Langmuir isotherm provided the best fit to a straight line and $\mathrm{R}_{2}$ value close to 1 (Figure 14). The equilibrium constant for the adsorption process $\left(\mathrm{K}_{\mathrm{ads}}\right)$ has a relation with the standard free energy of adsorption $\left(\Delta G_{\text {ads }}\right)$ which is represented by equation (12).

$$
\Delta G_{\mathrm{ads}}=-R T \ln \left(55.5 K_{\mathrm{ads}}\right)
$$

where 55.5 value is the molar water concentration whereas the $R$ and $T$ are the gas constant and absolute temperature respectively.

It has been published recently that the $\Delta G_{\text {ads }}$ value has great importance in determining the nature of the interaction between the inhibitor molecules and the metal surface. If this value is equal to or less than $-20 \mathrm{~kJ} \mathrm{~mol}^{-1}$, it suggests that the adsorption interaction is physisorption, while if the $\Delta G_{\text {ads }}$ value is approximately equal to or greater than $-40 \mathrm{~kJ}$ $\mathrm{mol}^{-1}$, the adsorption interaction is chemisorption [24]. The value of $\Delta G_{\text {ads }}$ obtained in this investigation is $40 \mathrm{~kJ} \mathrm{~mol}^{-1}$, which implies that the nature of adsorption interaction of the investigated compound on mild steel surface is generally chemisorption. Usually, the chemical interactions between the inhibitor and the surface of the metal are initiated by the decomposition reaction. Thus, the adsorption isotherms of the investigated inhibitor molecules on mild steel surface might be firstly by a physisorption process but the adsorption dominant model as proposed by the value of $\Delta G_{\text {ads }}$ can be categorized as chemisorption [25].

\subsection{Surface Characterization}

The SEM images for mild steel in the absence and presence of $\mathbf{8 P}$ are displayed in Figure 15. The SEM micrograph in the absence of the inhibitor shows deep corrosion (Figure 15a), resulting in a harsh and unsmooth mild steel surface in a $1 \mathrm{M}$ hydrochloric acid environment. 
On the other hand, upon addition of the investigated inhibitor, the mild steel surface is protected and looks smoother (Figure 15b), which enhances the inhibition performance.
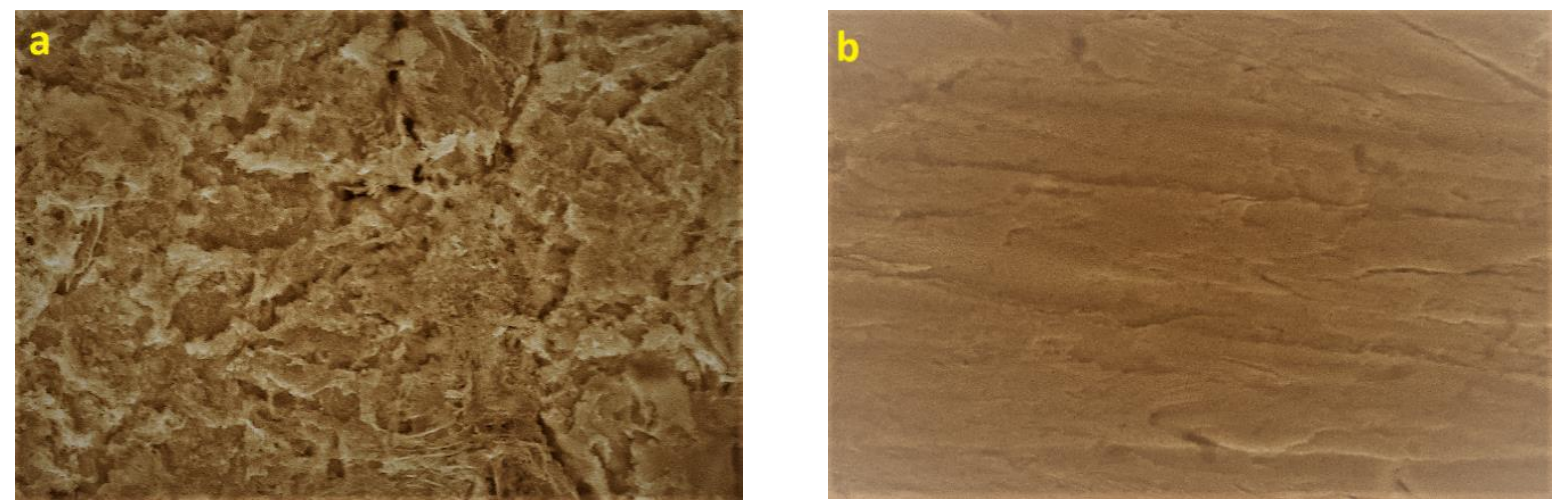

Figure 15. SEM images (a) in the absence (b) and presence of $5.0 \times 10^{-4} \mathrm{M} \mathbf{8 P}$.

\subsection{Quantum Chemical Calculations}

The optimized structure of the studied inhibitor molecules and the respective frontier molecular orbitals called HOMO and LUMO electron density surfaces of the investigated inhibitor are demonstrated in Figure 16. HOMO is the Highest Occupied molecular orbital, the highest-energy molecular orbital that has electrons in it. LUMO is the Lowest Unoccupied molecular orbital, the lowest-energy molecular orbital that does not have any electrons in it. The optimized structure in Figure 16 implies that the inhibitor molecules adopt near-planar geometry in which the $\mathrm{N}$-methylene groups in the piperazine and coumarin rings are only twisted. The near-planar geometry of the investigated inhibitor molecule may contribute to the high inhibitive efficiency due to the high planarity degree that was proved to support the best adsorption of the tested compound on the mild steel surface leading to an increase the inhibitive performance [26-28]. The highest occupied MO electron density surface suggests data about the most donating electron species of the inhibitor molecules to the favorable orbital of the acceptor sites. On the other hand, the lowest unoccupied MO electron density surface provides the active groups of the inhibitor molecules that possess accepting electrons with higher chances from donor sites.
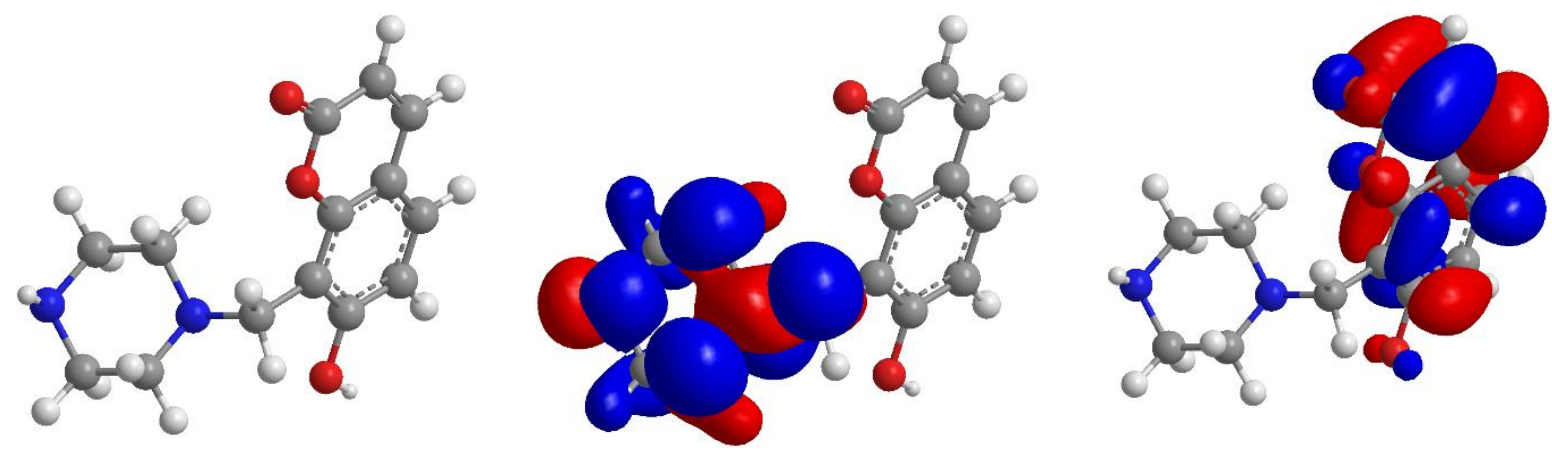

Figure 16. The optimum structure, HOMO and LUMO of $\mathbf{8 P}$. 
To define more about the HOMO and LUMO orbitals and their importance in predicting the nature of the interference between the molecules and the type of the interaction and bonds, wherein the redox reaction. In a redox reaction, the reductant has a HOMO with a high value and the oxidant has a LUMO with a low value, but the reductant HOMO is higher than the oxidant LUMO so that the electrons pass completely to the oxidant LUMO from the reductant HOMO (Figure 17). Predominantly the match of energy is poor, and hence no covalent bond is formed, just electrons are transferred. Sometimes a covalent bond is also formed. This depends on the energies of the orbitals, which is reflected by electronegativity.

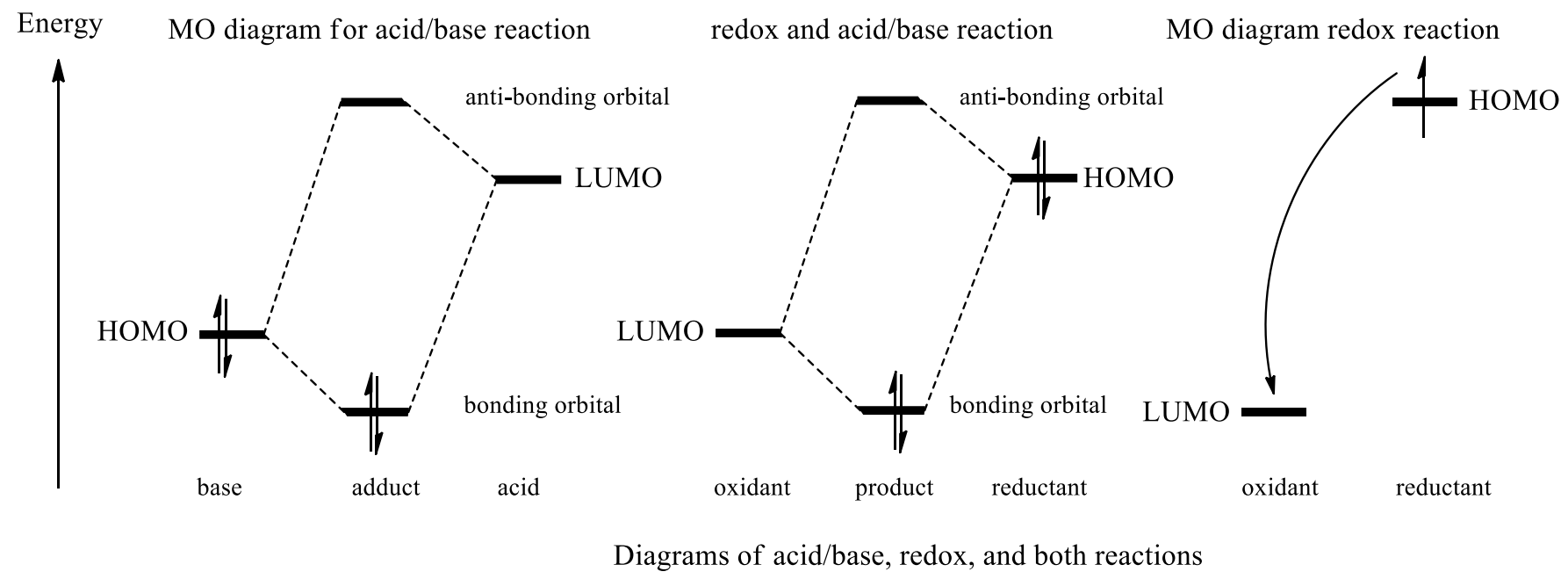

Figure 17. The HOMO and LUMO energies.

Molecules with a high LUMO, a low HOMO, and a big energy gap (HOMO-LUMO), such as hydrocarbons, are not quite reactive. Hydrocarbons do not react at room temperature.

The HOMO of the studied molecules is widely distributed over piperazine and is well delocalized over the methylene group but not the coumarin moiety, which does not contribute to the HOMO. The values of quantum chemical factors obtained for the investigated compound are displayed in Table 1. The energy of the highest occupied MO is the propensity measure of donating HOMO electrons of a molecule to the sufficient unoccupied orbital of the metal surface, so a molecule with high $E_{\mathrm{HO} O}$ value have the best ability to donate electrons [29]. The $E_{\mathrm{HOMO}}$ value of the investigated inhibitor was $-8.667 \mathrm{eV}$, which agrees with the experimental findings of inhibition efficiency. On the other hand, the $E_{\mathrm{LUMO}}$ is the affinity measure of the tested molecule to accept electrons, so that a low $E_{\mathrm{LumO}}$ value indicates the tendency of the inhibitor molecule to accept electrons from the d-orbitals of iron atoms. The findings of the energy of low unoccupied MOs in Table 1 shows a significant agreement with the tendency of the observed inhibitive efficiency, which proposes that the energy of low unoccupied MOs may be a significant descriptor for the relative inhibitive efficiency of the investigated inhibitor molecules. The energy gap $\left(\Delta E=E_{\mathrm{HOMO}}-E_{\mathrm{LUMO}}\right)$ is a considerable reactivity factor of inhibitor molecules for which a molecule with a low value of $\Delta E$ is generally more reactive and possesses higher inhibitive activity. The energy gap value 
obtained for the investigated inhibitor molecules is in good agreement with the inhibitive efficiency found in the experiments. The electronegativity, $\chi$, measures the propensity of an atom to attract a shared pair of electrons, and it is the index of reactivity which predicts the range to which an inhibitor molecule possesses their electrons. The electronegativity with a high value represents the lower electron donation chance of the molecule. The trend of electronegativity value obtained for the investigated inhibitor molecules is listed in Table 1, which proposes that the studied inhibitor molecules have the possibility of donating electrons to the d-orbital of iron atoms of the mild steel surface. The electronegativity trend is agreed with the inhibition efficiency results. The fraction of electrons transferred $(\Delta N)$ value is listed in Table 1 and represents the fraction of electrons transferred from the studied inhibitor molecules to the iron d-orbital atoms of the mild steel surface, also in agreement with the trend of the experimental results of inhibition efficiency. The dipole moment $(\mu)$ is used to relate the inhibition efficiency of an organic molecule to different perspectives. The first point of view is that an increase in the dipole moment of an organic molecule leads to a decrease in the efficiency of corrosion inhibition in corrosive solutions, with an explanation that a decrease in dipole moment supports the preference for the accumulation of the inhibitor molecules on the surface layer of the metal. The second point of view is, in general, the opposite of the first view, as it assumes that with a higher value of the dipole moment, the inhibition efficiency is enhanced due to the increase in dipole-dipole interactions between the damper molecules and the metal surface [30-33]. The dipole moment value obtained for the investigated corrosion inhibitor molecules is listed in Table 1, which agrees with the authors' opinion who consider that the high value of dipole moment leads to high inhibitive efficiency. The quantum chemical indexes (HOMO, LUMO, $\chi, \Delta N$ and $\mu$ ) that demonstrated significant agreement with the methodological findings are those that propose the increase of inhibitive efficiency for an inhibitor molecule with a considerable ability to electron donation. The practical application of this assumption is also based on the fact that the surface of mild steel in a corrosive environment is said to be filled with positive charges, such that the interaction of the electron donation inhibitor molecules with the positively charged mild steel surface is an appropriate process.

This indicates that the adsorption of the investigated corrosion inhibitor molecules on the surface of mild steel mostly occurs by donating the electron from the electronic highdensity atoms or active groups to the d-orbital of iron atoms.

The global hardness $(\eta)$ and global softness $(\sigma)$ are other significant parameters that give data about the stability of the molecule and its reactivity [34]. The hard molecule has a large energy gap, but the soft molecule has a low energy gap. Thus, the inhibitor molecules which have a low value of global hardness, and a high value of global softness propose a significant inhibition efficiency [35, 36]. 
Table 1. Quantum chemical parameters of the studied molecules.

\begin{tabular}{cc}
\hline$\Delta E=E_{\mathrm{HOMO}}-E_{\mathrm{LUMO}}$ & Value \\
\hline$\eta=-\left(\frac{E_{\mathrm{HOMO}}-E_{\mathrm{LUMO}}}{2}\right)$ & $-8.667+(-4.506)=-4.161 \mathrm{eV}$ \\
$\chi=-\left(\frac{E_{\mathrm{HOMO}}-E_{\mathrm{LUMO}}}{2}\right)$ & $2.805 \mathrm{eV}$ \\
$\Delta N=\frac{\chi_{\mathrm{Fe}}-\chi_{\mathrm{inh}}}{2\left(\eta_{\mathrm{Fe}}+\eta_{\mathrm{inh}}\right)}$ & $0.3565 \mathrm{eV}$ \\
$\mu$ & $6.414 \mathrm{eV}$ \\
\hline
\end{tabular}

\section{Conclusions}

A new umbelliferon derivative, namely 8P, was synthesized, and its structure was characterized and confirmed by Fourier transform infrared spectroscopy and nuclear magnetic resonance spectroscopy. $8 \mathrm{P}$ has been studied for its corrosion inhibitive performance on mild steel in $1 \mathrm{M}$ hydrochloric acid solution. The experimental findings demonstrated that the investigated inhibitor inhibited mild steel corrosion in the corrosive solution and the inhibition efficiency increased with increasing concentration. The experimental data revealed that the highest inhibition efficiency was $93.42 \%$ at $0.0005 \mathrm{M}$. Also, the inhibition efficiency increased with increasing immersion time. SEM micrographs demonstrated that the studied inhibitor molecules prevented the mild steel surface from having direct contact with the hydrochloric acid environment. The adsorption of corrosion inhibitor molecules on mild steel surface in a hydrochloric acid environment follows the Langmuir isotherm. The tendency of quantum chemical parameters such as energy gap, HOMO, LUMO, electronegativity, hardness, softness, and $\Delta N$ for the synthesized corrosion inhibitor is in good agreement with the inhibition efficiency.

\section{Acknowledgments}

This study was supported by the University of Technology, Baghdad, Iraq.

\section{Conflicts of Interest}

The authors declare no conflict of interest. 


\section{References}

1. F.G. Medina， J.G. Marrero, M. Macías-Alonso, M. González, I. Córdova-Guerrero, A.T. García and S. Osegueda-Robles, S. Coumarin heterocyclic derivatives: chemical synthesis and biological activity, Nat. Prod. Rep., 2015, 32, 1472-1507.

2. A. Lacy and R. O'Kennedy, Curr. Pharm. Des., 2004, 10, 3797-3811.

3. A. Fouda, M. Ismai, A. Abousalem and E. Elewady, Experimental and theoretical studies on corrosion inhibition of 4-amidinophenyl-2,2'-bifuran and its analogues in acidic media, $R S C A d v$., 2017, 7, 46414-46430. doi: 10.1039/C7RA08092A

4. K. Ostrowska, Coumarin-piperazine derivatives as biologically active compounds, Saudi Pharm J., 2020, 28, no. 2, 220-232. doi: 10.1016/j.jsps.2019.11.025

5. S.B. Al-Baghdadi, F.G. Hashim, A.Q. Salam, T.K. Abed, T.S. Gaaz, A.A. Al-Amiery, A.A.H. Kadhum, K.S. Reda and W.K. Ahmed, Synthesis and corrosion inhibition application of NATN on mild steel surface in acidic media complemented with DFT studies, Results Phys., 2018, 8, 1178-1184. doi: 10.1016/j.rinp.2018.02.007

6. K.F. Al-Azawi, I.M. Mohammed, S.B. Al-Baghdadi, T.A. Salman, H.A. Issa, A.A. AlAmiery, T.S. Gaaz and A.A.H. Kadhum, Experimental and quantum chemical simulations on the corrosion inhibition of mild steel by 3-((5-(3,5-dinitrophenyl)-1,3,4thiadiazol-2-yl)imino)indolin-2-one, Results Phys., 2018, 9, 278-283. doi: 10.1016/j.rinp.2018.02.055

7. H.R. Obayes, G.H. Alwan, A.H.MJ. Alobaidy, A.A. Al-Amiery, A.A.H. Kadhum and A.B. Mohamad, Quantum chemical assessment of benzimidazole derivatives as corrosion inhibitors, Chem. Cent. J., 2014, 8, no. 21, 1-8. doi: 10.1186/1752-153X-8$\underline{21}$

8. T.A. Salman, D.S. Zinad, S.H. Jaber, M. Shayaa, A. Mahal, M.S. Takriff and A.A. AlAmiery, Effect of 1,3,4 thiadiazole scafold on the corrosion inhibition of mild steel in acidic medium: an experimental and computational study, J. Bio Tribo-Corros., 2019, 5, no. 48, 1-11. doi: 10.1007/s40735-019-0243-7

9. D.S. Zinad, M. Hanoon, R.D. Salim, S.I. Ibrahim, A.A. Al-Amiery, M.S. Takriff and A.A.H. Kadhum, A new synthesized coumarin-derived Schiff base as a corrosion inhibitor of mild steel surface in hcl medium: Gravimetric and dft studies, Int. J. Corros. Scale Inhib., 2020, 9, no. 1, 228-243.

10. D.S. Zinad， Q.A. Jawad， M.A.M. Hussain， A. Mahal， L. Mohamed and A.A. AlAmiery, Adsorption, temperature and corrosion inhibition studies of a coumarin derivatives corrosion inhibitor for mild steel in acidic medium: Gravimetric and theoretical investigations, Int. J. Corros. Scale Inhib., 2020, 9, no. 1, 534-151. doi: 10.17675/2305-6894-2020-9-1-14

11. M.M. Hanoon, A.M. Resen, A.A. Al-Amiery, A.A.H. Kadhum and M.S. Takriff, Theoretical and Experimental Studies on the Corrosion Inhibition Potentials of 2-((6Methyl-2-Ketoquinolin-3-yl)Methylene) Hydrazinecarbothioamide for Mild Steel in $1 \mathrm{M} \mathrm{HCl}$, Prog. Color Colorants Coat., 2022, 15, 21-33. 
12. S. Junaedi, A.A.H. Kadhum, A. Al-Amiery, A.B. Mohamad and M.S. Takriff, Synthesis and characterization of novel corrosion inhibitor derived from oleic acid: 2-Amino-5Oleyl-1,3,4-Thiadiazol (AOT), Int. J. Electrochem. Sci., 2012, 7, 3543-3554.

13. A.A. Al-Amiery, Y.K. Al-Majedy, A.A.H. Kadhum and A.B. Mohamad, Synthesis of new coumarins complemented by quantum chemical studies, Res. Chem. Intermed., 2016, 42, no 4, 3905-3918.

14. A.Y.I. Rubaye, K.S. Rida, A.Q. Salam and A. Al-Amiery, Acetamidocoumarin as a based eco-friendly corrosion inhibitor, Int. J. ChemTech Res., 2016, 9, 39-47.

15. A.A. Al-Amiery, Y.K. Al-Majedy, A.A.H. Kadhum and A.B. Mohamad, New coumarin derivative as an eco-friendly inhibitor of corrosion of mild steel in acid medium, Molecules, 2015, 20, no. 1, 366-383. doi: 10.3390/molecules20010366

16. A.A.H. Kadhum, A.B. Mohamad, L.A. Hammed, A.A. Al-Amiery, N.H. San and A.Y. Musa, Inhibition of mild steel corrosion in hydrochloric acid solution by new coumarin, Mater., 2014, 7, no. 6, 4335-4348. doi: 10.3390/ma7064335

17. A.B. Mohamad, A.A.H. Kadhum, A.A. Al-Amiery, L.C. Ying and A.Y. Musa, Synergistic of a coumarin derivative with potassium iodide on the corrosion inhibition of aluminum alloy in $1.0 \mathrm{M} \mathrm{H}_{2} \mathrm{SO}_{4}$, Met. Mater. Int., 2014, 20, 459-467. doi: 10.1007/s12540-014-3008-3

18. N.K. Gupta, C. Verma, R. Salghi, H. Lgaz, A.K. Mukherjee and M.A. Quraishi, New phosphonate based corrosion inhibitors for mild steel in hydrochloric acid useful for industrial pickling processes: experimental and theoretical approach, New J. Chem., 2017, 41, 13114-13129. doi: 10.1039/C7NJ01431G

19. C. Wang, C. Lai, B. Xie, X. Guo, D. Fu, B. Li and S. Zhu, Corrosion inhibition of mild steel in $\mathrm{HCl}$ medium by S-benzyl-O, $\mathrm{O}^{\prime}$-bis(2-naphthyl)dithiophosphate with ultra-long lifespan, Results Phys., 2018, 10, 558-567. doi: 10.1016/j.rinp.2018.07.002

20. A.A. Al-Amiery, L.M. Shaker and A. Kadhum, Corrosion inhibition of mild steel in strong acid environment by 4-((5,5-dimethyl-3-oxocyclohex-1-en-1yl)amino)benzenesulfonamide, Tribology in industry, 2020, 42, no. 1, 89-101. doi: 10.24874/ti.2020.42.01.09

21. A. Al-Amiery, A. Salman, T. Alazawi, K. Shaker, L. Kadhum and A. Takrif, Quantum chemical elucidation on corrosion inhibition efficiency of Schiff base: DFT investigations supported by weight loss and SEM techniques, Int. J. Low-Carbon Technol., 2020, 15, 202-209.

22. S.S. Al-Taweel, K.W.S. Al-Janabi, H.M. Luaibi, A.A. Al-Amiery and T.S. Gaaz, Evaluation and characterization of the symbiotic effect of benzylidene derivative with titanium dioxide nanoparticles on the inhibition of the chemical corrosion of mild steel, Int. J. Corros. Scale Inhib., 2019, 8, no. 4, 1149-1169. doi: 10.17675/2305-6894-2019$\underline{8-4-21}$

23. A. Kadhim, A.A. Al-Amiery, R. Alazawi, M.K.S. Al-Ghezi and R.H. Abass, Corrosion inhibitors. A review, Int. J. Corros. Scale Inhib., 2021, 10, no. 1, 54-67. doi: $\underline{10.17675 / 2305-6894-2021-10-1-3}$ 
24. P. Singh, E. Ebenso, L. O. Olasunkanmi, I.B. Obot and M.M. Quraishi, Electrochemical, theoretical, and surface morphological studies of corrosion inhibition effect of green naphthyridine derivatives on mild steel in hydrochloric acid, J. Phys. Chem. C, 2016, 120, 3408-3419. doi: $10.1021 /$ acs.jpcc.5b11901

25. S. Junaedi, A. Al-Amiery, A. Kadihum, A.H. Kadhum and A. Mohamad, Inhibition effects of a synthesized novel 4-Aminoantipyrine derivative on the corrosion of mild steel in hydrochloric acid solution together with quantum chemical studies, Int. J. Mol. Sci., 2013, 14, 11915-11928. doi: 10.3390/ijms140611915

26. A. Alobaidy, A. Kadhum, S. Al-Baghdadi, A. Al-Amiery, A. Kadhum, E. Yousif et al., Eco-friendly corrosion inhibitor: experimental studies on the corrosion inhibition performance of creatinine for mild steel in $\mathrm{HCl}$ complemented with quantum chemical calculations, Int. J. Electrochem. Sci., 2015, 10, 3961-3972.

27. T. Arslan, F. Kandemirli, E. Ebenso, I. Love and H. Alemu, Quantum chemical studies on the corrosion inhibition of some sulphonamides on mild steel in acidic medium, Corros. Sci., 2009, 51, 35-47.

28. F. Bentiss and M. Lagrenee, Heterocyclic compounds as corrosion inhibitors for mild steel in hydrochloric acid medium-correlation between electronic structure and inhibition efficiency, J. Mater. Environ. Sci., 2011, 2, 13-17.

29. K. Ansari and M. Quraishi, Experimental and computational studies of naphthyridine derivatives as corrosion inhibitor for N80 steel in 15\% hydrochloric acid, Phys. E, 2015, 69, 322-331.

30. N. Soltani, M. Behpour, E. Oguzie, M. Mahluji and M. Ghasemzadeh, Pyrimidine-2thione derivatives as corrosion inhibitors for mild steel in acidic environments, $R S C A d v$., 2015, 5, 11145-11162.

31. E. E. Oguzie, C. Enenebeaku, C. Akalezi, S.C. Okoro, A. Ayuk and E. Ejike, Adsorption and corrosion-inhibiting effect of dacryodis edulis extract on low-carbon-steel corrosion in acidic media, J. Colloid Interface Sci., 2010, 349, 283-292.

32. A. Kadhim, A.K. Al-Okbi, D.M. Jamil, A. Qussay, A.A. Al-Amiery, T.S. Gaas, A.A.H. Kadhum, A.B. Mohamad and M.H. Nassir, Experimental and theoretical studies of benzoxazines corrosion inhibitors, Results Phys., 2017, 7, 4013-4019. doi: 10.1016/j.rinp.2017.10.027

33. H.R. Obayes, A.A. Al-Amiery, G.H. Alwan, T.A. Abdullah, A.A.H. Kadhum and A.B. Mohamad, Sulphonamides as corrosion inhibitor: experimental and DFT studies, J. Mol. Struct., 2017, 1138, 27-34. doi: 10.1016/j.molstruc.2017.02.100

34. D.M. Jamil, A.K. Al-Okbi, S.B. Al-Baghdadi, A.A. Al-Amiery, A. Kadhim and T.S. Gaaz, Experimental and theoretical studies of Schiff bases as corrosion inhibitors, Chem. Cent. J., 2018, 12, no. 7, 1-7. doi: 10.1186/s13065-018-0376-7

35. D. Mahmood, A.K. Al-Okbi, M.M. Hanon, K.S. Rida, A.F. Alkaim, A.A. Al-Amiery, A. Kadhum and A.A.H. Kadhum, Carbethoxythiazole corrosion inhibitor: as an experimentally model and DFT theory, J. Eng. Appl. Sci., 2018, 13, 3952-3959. doi: $\underline{10.3923 / \text { jeasci.2018.3952.3959 }}$ 
36. L.O. Olasunkanmi, I.B. Obot, E.E. Ebenso, Adsorption and corrosion inhibition properties of $\mathrm{N}$-\{n-[1-R-5-(quinoxalin-6-yl)-4,5-dihydropyrazol-3-yl]phenyl $\}$ methane sulfon amides on mild steel in $1 \mathrm{M} \mathrm{HCl}$ : experimental and theoretical studies. RSC $A d v$., 2016, 6, 86782-86797. doi: 10.1039/C6RA11373G

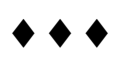

